\title{
BONPLAND, SESQUICENTENARIO DE SU MUERTE
}

Conferencia pronunciada el 16 de mayo de 2008 en el Museo Bonpland de la ciudad de Corrientes, en el acto de homenaje a Aimé Bonpland auspiciado por la Academia Nacional de Ciencias de Córdoba y la Facultad de Ciencias Agrarias de la UNNE

\section{ANTONIO KRAPOVICKAS}

Llevan el nombre de Bonpland poblaciones en Corrientes, Misiones y Venezuela. Es nombre de calles en Montevideo, Buenos Aires, Rosario, Córdoba, Bahía Blanca, Posadas, Puerto Iguazú, Corrientes. Paso de los Libres, Curuzú Cuatiá. En Buenos Aires hay un «Mercado Bonpland». En Oberá hay una escuela. Hay un cerro en Venezuela, otro en Misiones. Hay un río en Tierra del Fuego. Dos revistas botánicas llevan su nombre: Bonplandia. Le dedicaron los géneros Bonplandia Cav. (1800), Bonplandia Willd. (1802) y Bomplandiella Speg. (1886).Una comparsa del Carnaval de Paso de los Libres lo tuvo como motivo. Este año otra comparsa, Sapucay, usó su vida como tema y obtuvo el primer premio en el carnaval de Corrientes. Por añadidura, el año pasado una comparsa del carnaval carioca, tuvo como motivo al barón von Humboldt.

Aimé Jacques Alexandre Goujaud Bonpland (Fig. 1) nació en La Rochelle, en la costa atlántica

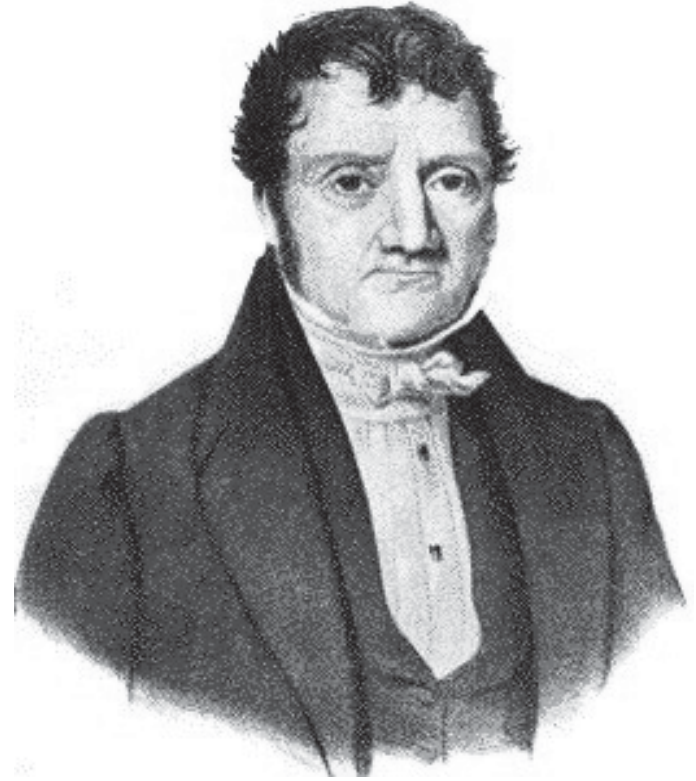

Fig. 1. Aimé Bonpland a los 64 años. Litografía sobre un dibujo a lápiz hecho por Carlos E. Pellegrini el 3 de enero de 1937 en la casa de Pedro de Angelis en Buenos Aires. de Francia el 28 de agosto de 1773, donde su padre ejercía la medicina y ya llevaba el apodo de Bonpland. Era hijo y hermano de médicos.

A los 18 años se traslada a París para estudiar medicina y donde también se dedica a la Botánica. Tuvo maestros como de Jussieu Lamarck y de Candolle, cuando se estructuraban los sistemas naturales de clasificación y se comienza a esbozar la teoría de la evolución, publicada por Lamarck en 1809.

En París se encuentra con el barón von Humboldt, notable naturalista de formación universal, con quién establece una larga amistad.

Resultado de esta amistad fue su participación en el gran viaje de estudio y descubrimiento a la América hispana.

Federico Enrique Alejandro, Barón von Humboldt nació en Berlin, su padre fue compañero de armas de Federico el Grande, rey de Prusia, su madre era una hugonote francesa cuya familia se refugió en Alemania después de la revocación del edicto de Nantes en 1685. Su hermano Car-

${ }^{1}$ Instituto de Botánica del Nordeste, Casilla de Correo 209, 3400 Corrientes, Argentina. 
los Guillermo fue un gran filólogo clásico e iniciador del estudio comparado de las lenguas.

En el momento de su viaje a América hispana, poco se sabía en Europa de la flora de América (Figs. 2 y 3).

\begin{tabular}{ll}
\hline NE de Brasil & Piso \& Marcgraf (1637-1642) \\
México & F. Hernández (1615, 1651) \\
Antillas & Plumier (1693) \\
& Sloane (1696, 1707, 1725) \\
& Swartz (1788-1889) \\
& Richard (1781-1889) \\
Chile & Ovalle (1646) \\
& Molina (1782) \\
Venezuela & Loefling (1758) \\
Perú & Ruiz \& Pavón (1794) \\
\hline
\end{tabular}

Fig. 2. Lo publicado antes del viaje de Humboldt y Bonpland (1799-1804).

\begin{tabular}{ll}
\hline Perú & de Jussieu (La Condamine) (1735-1771) \\
Colombia & Mutis (L.f. 1781) \\
Puertos & Commerson (Bougainville) (1767) \\
& Banks \& Solander (Cook) (1768) \\
& Neé \& Haenke (Malespina) (1789-1794) \\
\hline
\end{tabular}

Fig. 3. Colecciones anteriores a Humboldt y Bonpland (1799-1804).

Bonpland tiene 26 años y Humboldt 30 cuando embarcan en La Coruña rumbo a América. Es interesante comparar las edades de viajeros contemporáneos a Bonpland (Fig. 4).

Este viaje es considerado el "segundo descubrimiento de América", pero esta vez desde el punto de vista científico.

Humboldt hizo observaciones astronómicas, geográficas, meteorológicas, etnográficas, biológicas, geológicas y paleontológicas. Bonpland se dedicó principalmente a las colecciones botánicas.

Exploraron Venezuela, Colombia, Ecuador, Perú (hasta Lima), las fuentes de los ríos Orinoco y Amazonas, México y Cuba.

El método de trabajo de Bonpland consistió en estudiar cada ejemplar coleccionado «in situ», efectuar una detallada descripción y

\begin{tabular}{ll}
\hline Edad & \multicolumn{1}{c}{ Viajeros } \\
\hline 22 & Darwin - Pilger \\
23 & Martius - Langsdorff \\
24 & d' Orbigny - Poeppig - Gardner - Weddell \\
25 & Sellow \\
26 & Bonpland \\
27 & Marggraf \\
28 & $\begin{array}{l}\text { Gay - Gaudichaud - Ferreira, Alexandre } \\
\text { Rodrigues - Malme }\end{array}$ \\
30 & Humboldt - Miers \\
\hline
\end{tabular}

Fig. 4. Edades de viajeros contemporáneos de Bonpland.

anotar datos sobre el lugar. No volvía a coleccionar ninguna de las especies ya estudiadas. Es por ello que el herbario con unos 6000 ejemplares contiene más de 4500 especies nuevas y para cada especie casi siempre citan por lo común un solo ejemplar. Este era el método utilizado por los grandes viajeros y lo muestra el contenido de los herbarios históricos. Así son los herbarios de Linneo, de Candolle, Lamarck, de Jussieu, etc.: un solo ejemplar por especie, raro dos. En esa época interesaba el «sistema», o sea ordenar el conjunto.

A la colección de plantas la dividieron en 3 partes: una de Humboldt, que donó al Jardin des Plantes, consta de unos 6000 ejemplares y se mantiene aparte (PHU), la segunda correspondió a Bonpland, la que ahora se encuentra incorporada al herbario general de París, y la tercera forma parte del Herbario Willdenow de Berlín.

Duplicados de esta colección se encuentran en: B, CGE, F, FI, G, HAL, KIEL, L, LINN, LR, MEDELL, P, PC, PJU y NY.

Regresan a Francia el 3 de agosto de 1804, año en que Napoleón se corona Emperador (Fig. 5).

El 13 de marzo de 1805, por un decreto imperial se acepta la donación de la parte de Humboldt del gran herbario americano y se le concede a Bonpland una pensión anual de 3000 francos. 
1773, 28-VIII, Nace Bonpland en La Rochelle

1799, 5-VI, Humboldt y Bonpland embarcan en La

Coruña. 26 años

1800, Bonplandia Cavanilles

1802, Bonplandia Willdenow

1804, 3-VIII, Regresan a Francia

1804, Napoleón se corona Emperador.

1808, 3-VIII, Josefina contrata a Bonpland como administrador de La Malmaison.

1809, Josefina se divorcia.

1813, Kunth comienza a estudiar las plantas

1814, Josefina muere

1814. Muere Mutis

1814, Napoleón abdica

1815, Waterloo

Fig. 5. Cronología.

En 1805 comienzan a publicar los primeros fascículos de Plantes équinoxiales, que terminan en 1808, año en que Napoleón invade España.

Tanto los viajes como las lujosas publicaciones de los resultados fueron pagados por Humboldt con fondos propios, invirtiendo en ello toda su fortuna. Tanto es así que Humboldt terminó sus días con una pensión otorgada por su ciudad.

El 3 de agosto de 1808 la emperatriz Josefina contrata a Bonpland como intendente de la Malmaison.

En 1813 Carol Sigysmund Kunth un joven de 25 años comienza a estudiar las plantas de las colecciones de Humboldt y Bonpland.

En 1814 abdica Napoleón y en 1815 la batalla de Waterloo decide su alejamiento definitivo de Francia.

En 1814 muere Josefina y Bonpland, sospechoso bonapartista, deja su cargo en la Malmaison.

Sobre la base de sus colecciones y de sus descripciones se publicó el Nova genera et species plantarum, de Humboldt, Bonpland y Kunth, en 7 gruesos volúmenes, entre 1815 y 1825, donde se describen unas 4500 especies nuevas.

Con el comienzo de esta publicación Bonpland abandona su participación en la redacción final del texto, pero queda clara su colaboración con sus notas y descripciones a campo.

Decide volver a América. Recibe un ofrecimiento de Bolívar para continuar los estudios de Mutis en Colombia, pero la suerte adversa de las tropas de Bolívar hacen fracasar esta posibilidad.

Con la caída de Napoleón y la suspensión del bloqueo, Bonpland hace varios viajes a Londres donde se contacta con Rivadavia, Sarratea y Belgrano, quienes lo invitan a viajar y residir en el Río de la Plata.

Llega a Buenos Aires en febrero de 1817, cuando la batalla de Chacabuco anuncia el comienzo de la campaña sanmartiniana. Tiene 44 años. Instala un jardín botánico en lo que es hoy la plaza Garay, cerca de Constitución. Colecciona en las islas del Paraná, en la isla Martín García, donde encuentra unas pocas plantas de yerba mate (Fig. 6).

\begin{tabular}{|c|c|c|c|}
\hline 1817, II, & Buenos Aires & 44 & Chacabuco \\
\hline \multirow[t]{2}{*}{ 1820, X, } & Corrientes & & $\begin{array}{l}\text { IX, Artigas cruza a } \\
\text { Paraguay }\end{array}$ \\
\hline & & & $\begin{array}{l}\text { XI, Republica de } \\
\text { Entre Ríos }\end{array}$ \\
\hline \multirow[t]{2}{*}{ 1821, VI, } & Santa Ana & & $\begin{array}{l}\text { Provincia Cisplatina } \\
\text { de Brasil }\end{array}$ \\
\hline & & & $\begin{array}{l}\text { Universidad de } \\
\text { Buenos Aires }\end{array}$ \\
\hline 1821, XII, & capturado & 48 & \\
\hline 1826-33 & & & Viaje de Saint Hilaire \\
\hline $1827-28$ & & & d'Orbigny, Corrientes \\
\hline 1828-32 & & & Gay, Chile \\
\hline 1827 & & & Batalla de Ituzaingó \\
\hline 1828 & & & Rep. Or. del Uruguay \\
\hline 1831, II, & sale de Itapúa & 57 & \\
\hline
\end{tabular}

Fig. 6. Cronología.

No tiene idea de que se metió en un berenjenal. Los sucesos históricos se desenvuelven en forma caótica.

En octubre de 1820 viaja a Corrientes. Ese mismo año Artigas, vencido, cruza al Paraguay donde pasará sus últimos años y Ramírez crea la República de Entre Ríos, la que abarcaba Entre Ríos, Corrientes y Misiones y pretendía incluir también a Paraguay y Uruguay. 
En Corrientes Bonpland se encuentra con el General Ramírez quién le ofrece un gran apoyo a su proyecto colonizador.

En junio de 1821 llega Bonpland a Misiones y se instala en la antigua reducción jesuítica de Santa Ana, donde encuentra yerbales abandonados, los cuales limpia y pone en condiciones de explotación.

En ese año 1821 muere Ramírez decapitado, cuando el General Estanislao López, Gobernador de Santa Fé, cambia de bando gracias al aporte de 30000 vacas que provee el estanciero bonaerense Juan Manuel de Rosas.

En ese mismo año Brasil completa la ocupación del Uruguay y crea la provincia Cisplatina.

También en ese año San Martín es proclamado Protector del Perú y se crea la Universidad de Buenos Aires.

En diciembre de (1821) Bonpland es capturado por orden del Dictador José Gaspar Rodríguez de Francia. Sólo 6 meses estuvo Bonpland en Santa Ana. Hay varias explicaciones de esta actitud de Francia. Suponía que era un espía. Al explotar los yerbales competiría con el monopolio que los paraguayos tenían del comercio de la yerba. Se instaló en un territorio que Francia consideraba paraguayo y en la única ruta de comunicación que tenía el Paraguay con el exterior.

El 2 de febrero de 1831 sale del Paraguay por Itapuá, después de 9 años y dos meses de reclusión. En Paraguay residió en el Cerrito entre las antiguas misiones jesuíticas de Santa María y Santa Rosa en el sur del país, donde podía moverse libremente dentro de un radio de varias leguas. Durante su reclusión llegó a ser un rico cultivador, en su propiedad tenía 45 personas empleadas.

"He dejado en el Paraguay un establecimiento agrícola bien montado; cultivé algodón, caña de azúcar, maní, cinco especies de mandioca, varias especies de batata, la planta de la yerba, tenía establecidas plantaciones de vid, naranjos y varias especies del género Citrus, guayabos, etc. También he dejado una destilería, un aserradero, una herrería y un hospital compuesto de cuatro habitaciones, donde tenía constantemente enfermos. A todo esto debo agregar cuatrocientas vacas, bueyes, mulas y caballos para hacer marchar con faci- lidad mi establecimiento".

Cuando deja Itapuá, cruza el río Paraná con todo su ganado y con 8 carretas con sus pertenencias. Se dirije a São Borja, donde se instala en sus proximidades a orillas del Ayo. Piratiní. Tiene 57 años (Fig. 7).

\begin{tabular}{|c|c|}
\hline 1831, II, & San Borja \\
\hline 1832 & $\begin{array}{l}\text { Envía } 25 \text { cajas: herbario, pájaros, objetos de } \\
\text { hist. natural }\end{array}$ \\
\hline $1835-45$ & $\begin{array}{l}\text { Rep. Rio Grande do Sul, Guerra de los } \\
\text { Farapos }\end{array}$ \\
\hline 1838 & Santa Ana, enfiteusis \\
\hline 1839 & Pago Largo $\left(\mathbf{1}^{\mathbf{0}}\right)$ \\
\hline \multirow[t]{2}{*}{1840} & Muere Gaspar de Francia \\
\hline & Campaña de Lavalle $\left(2^{\circ}\right)$ \\
\hline \multirow[t]{2}{*}{1841} & Muere Lavalle \\
\hline & Batalla de Caaguazú $\left(3^{\circ}\right)$ \\
\hline \multirow[t]{2}{*}{1843} & $\begin{array}{l}\text { Madariaga funda Paso de los Libres y } \\
\text { muere en él }\end{array}$ \\
\hline & Rincón de Vences $\left(4^{\circ}\right)$ \\
\hline $1850-52$ & Campaña de Caseros $\left(5^{\circ}\right)$ \\
\hline
\end{tabular}

Fig. 7. Cronología.

Recibe varias ofertas para volver a Europa pero Bonpland prefiere quedarse en América. Recibe ofrecimientos de Buenos Aires, que también rechaza.

En São Borja decide su futuro y lo manifiesta con estas palabras; "Habituado a vivir al aire libre, a la sombra de los árboles seculares de América, a oir el canto de los pájaros que suspenden sus nidos sobre mi cabeza, a sentarme para ver correr a mis pies las puras aguas de un arroyo; en lugar de todos estos dones, que encontraría yo en el barrio más aristocrático y brillante de París?. Encerrado en mi gabinete estaría obligado a trabajar día y noche por cuenta de un librero, tendría por toda compensación el placer de ver eclosionar de tiempo en tiempo en mis solapas una mezquina roseta. Perdería lo que yo más quiero, mi sociedad de predilección, mis plantas que hacen mi alegría y mi vida. No, no, es aquí donde debo vivir y morir".

Eligió para vivir lugares con el mismo paisaje que lo rodeó durante su cautiverio en Paraguay. Una combinación de campo, bos- 
que y agua, que es lo mismo que caracteriza a las reducciones jesuíticas. Esta situación la encontró en São Borja y en Santa Ana a ambas orillas del río Uruguay, dos de los pasos para cruzar este río.

En 1832 llega a Buenos Aires de donde envía a París 25 cajas con el herbario general, pájaros y otros objetos de historia natural.

En 1838 recibe en enfiteusis un campo de 5 leguas en el paso de Santa Ana, cerca de Uruguaiana.

Si cuando llegó al Plata se metió en un berenjenal, aquí se instaló en un tembladeral. Sus dos residencias a ambos lados del río Uruguay le permitían tranquilidad cuando la situación empeoraba.

Lo rodearon episodios bélicos a ambos lados del río. Entre 1835-1845 se crea la República de Río Grande do Sul, donde se desarrolla la guerra de los farapos. Entre 1839 y 1850 pasan cerca de su establecimiento 5 campañas militares, algunas muy sangrientas.

Después de la batalla de Pago Largo, el 31 de marzo de 1839, su estancia es saqueada, es despojado de 5000 ovejas, 200 caballos, 500 vacas, 400 burras, etc.

\begin{tabular}{ll}
\hline 1853 & Se traslada definitivamente a Santa Ana \\
1854, X, & Se crea el Museo o Exposición Provincial \\
1856, XI, & $\begin{array}{l}\text { Recibe en propiedad el establecimiento } \\
\text { de Santa Ana }\end{array}$ \\
1857, I, & Llega Burmeister a Argentina \\
1858, 11-V, & Fallece Bonpland a los 86 años
\end{tabular}

Fig. 8. Cronología.
Escribió mucho, en su diario de colecciones y en su correspondencia, pero publicó poco. Sus publicaciones las realizó en Europa (Fig. 9). En los países del Plata sólo dejó manuscritos, de éstos algunos pasaron a París y otros quedaron en manos de sus hijos, los cuales en su mayoría se conservan en el Museo J. A. Domínguez de la Facultad de Farmacia y Bioquímica de la Universidad de Buenos Aires.

Murió el 11 de mayo 1858 en su establecimiento de Santa Ana, acompañado por su hija, a la edad de 86 años.

En vida Bonpland recibió numerosos homenajes que muestran el gran respeto que supo ganar (Fig. 10).

De sus manuscritos los más importantes
1805. Pensión de 3000 fr. anuales
1827. Ciudadano honorario de México
1930. Miembro correspondiente del Museo Real de Hist. Nat., París
1833. Miembro de la Legión de Honor, Francia
1848. Caballero de la Legión de Honor, Emperador de Brasil 1852. "Bonplandia", revista Academia Leopoldina Carolina
1852. Miembro de la Academia Francesa de Ciencias
1853. Miembro, Logia "Cordialidade no Oriente", São Borja
1853. Miembro, Sociedad de Geografía, Francia
1853. Miembro, Sociedad de Medicina Montevideana
1854. Condecorado, Orden del Águila Roja, Rey de Prusia
1856. Doctor Honoris Causa, Universidad de Greifswald
1857. Miembro, Academia Leopoldina Carolina, Hannover

Fig. 10. Homenajes en vida.

Humboldt \& Bonpland, Essai sur la géographie des plantes (1805)

Bonpland, Claytonia cubensis Bonpl., Ann. Mus. Par. 7 (1806): 82. t. 6

Bonpland, Hebeandra, nouveae genre de plantes. Mag. Neuesten Entdeck. Gesammten Naturk. Ges. Naturf. Freunde Berlin 2: 40. (1808)

Humboldt \& Bonpland, Plantes équinoxiales, 1(1808), 1-234, 68 táb., 2(1809), 1-191, 75 táb.

Humboldt \& Bonpland, Monographia Melastomacearum, 1(1806-1816), 1-142, 60 táb., 2(1806-1823), 1-158, 60 táb.

Bonpland, Description des plantes rares cultivées à Malmaison et à Navarre, (1812-1817), 1-257, 64 táb.

Humboldt, Bonpland \& Kunth, Nova genera et species plantarum. 1(1815), 1-302, 94 táb.; 2(1817), 1-324, 95-192 táb.; 3(1818), 1-456, 193-300 tab.; 4(1820), 1-247, 301-412 tab.; 5(1821), 1-338, 413-505 tab.; 6(1823), 1-422, 513-600 tab.; 7(1825), 1-399, 601-700 tab.

Fig. 9. Lo publicado por Bonpland en Europa. 
son sus Diarios botánicos, donde a partir de su llegada a Buenos Aires en febrero de 1817, describe 2884 plantas que coleccionó durante su residencia en los países del Plata. Cada una de estas plantas las estudia en el lugar y realiza una detallada descripción, muchas veces acompañada con dibujos que muestran detalles específicos. Los últimos números son de Santa Ana en diciembre de 1857, poco antes de su muerte (Fig. 11).

Esta figura muestra una página de su diario botánico, en la que se describen dos especies ,

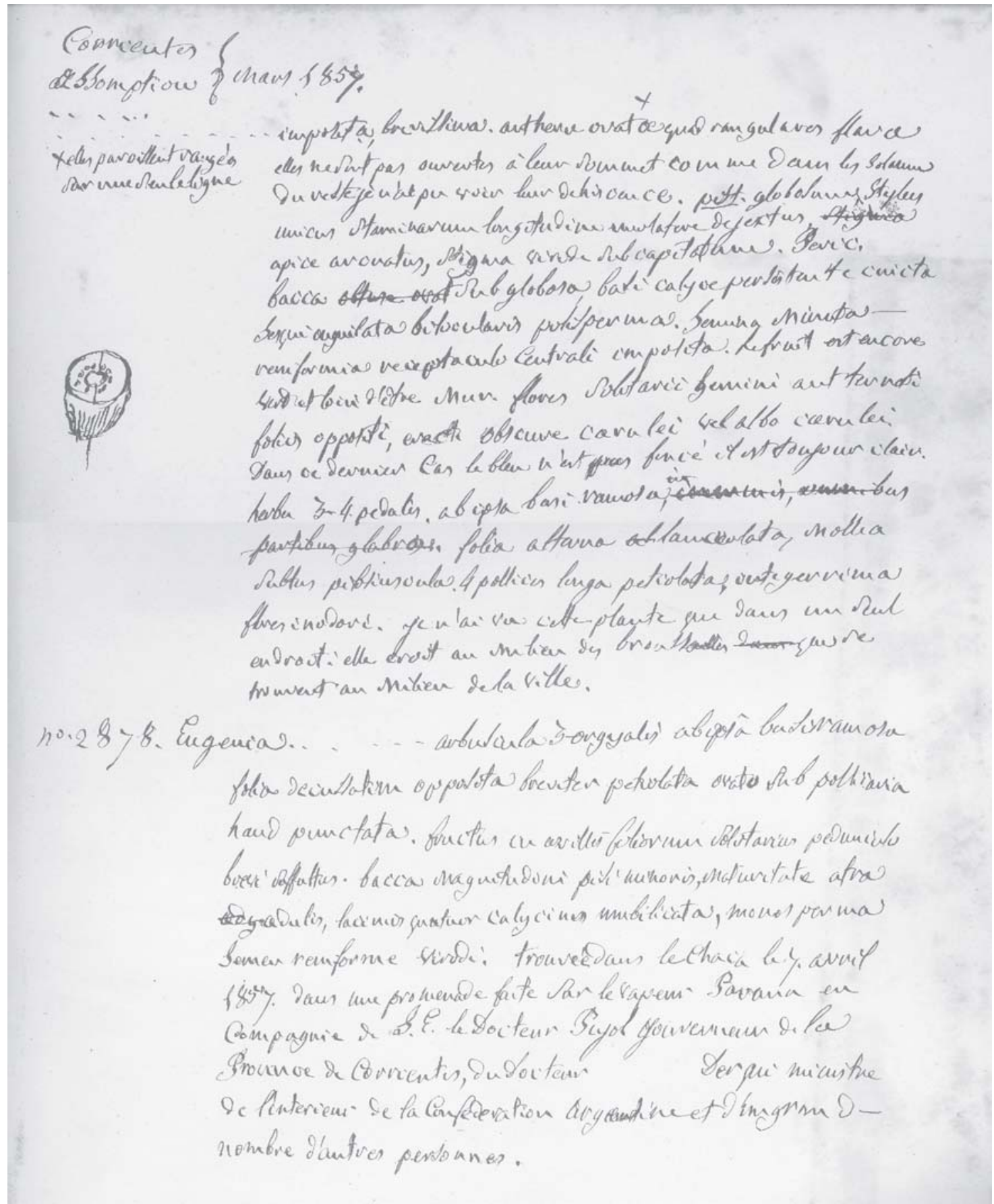

Fig. 11. Página de su Diario botánico. 
la segunda, una Eugenia, termina con una frase que pinta la estampa de don Amado Bonpland (Fig. 12).

"Encontrada en el Chaco el 7 de abril de 1857, durante un paseo en el vapor Paraná, en compañía del Dr. Pujol, gobernador de la provincia de Corrientes y del Dr. Derqui, ministro del interior de la Confederación Argentina".
Continúa su entusiasmo por las plantas y mantiene relación amistosa con prohombres de nuestra historia como los gobernadores Ferré y Pujol, el General Paz, el general Urquiza y tantos otros.

Supo ganar el afecto y el respeto de sus contemporáneos gracias a su condición bien ganada de hombre de bien.

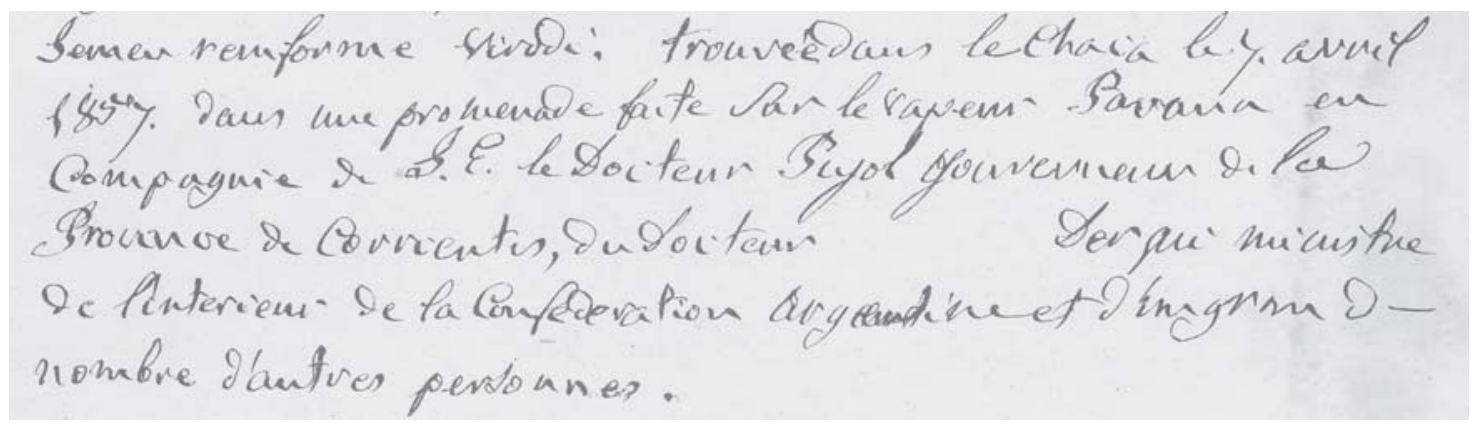

Fig 12. Último párrafo de la página anterior (Fig. 11). 
BONPLANDIA 17(1): 5-11. 2008 